\title{
GUBERNAMENTALIDAD Y BIOPOLÍTICA: UNA APROXIMACIÓN CON LOS SABERES Y PRÁCTICAS HISTÓRICAS DE LA TERAPIA OCUPACIONAL EN CHILE
}

\author{
GOVERNMENTALITY AND BIOPOLITIC: AN APPROACHTO KNOWLEDGE AND \\ HISTORICAL PRACTICES OF OCCUPATIONAL THERAPY IN CHILE
}

\section{Marcela Herrera Sandoval ${ }^{1}$, Cristián Valderrama Núñez ${ }^{2}$}

\begin{abstract}
Resumen:
Esta investigación muestra la relación existente entre los aspectos sociopolíticos del Estado, y la Terapia Ocupacional en Chile desde una perspectiva genealógica de análisis; desde esta forma de análisis se explica cómo se reproduce esta relación en los saberes y prácticas de la Terapia Ocupacional (TO).

Se considera como marco conceptual de análisis del ejercicio del poder, dos conceptos desarrollados por el autor Michel Foucault: la gubernamentalidad y biopolítica. La TO en este contexto, es definida como un dispositivo disciplinar, que correspondería a un instrumento del ejercicio del poder de las anteriores.

La metodología corresponde a un análisis genealógico. Se realizó un análisis de discurso desde una perspectiva Foucaulteana, a partir de 5 entrevistas semiestructuradas realizadas a Terapeutas Ocupacionales. Conjuntamente se analizaron, bajo la misma metodología, textos históricos de la disciplina en Chile. La unidad de análisis fueron las fuerzas políticas.

Las conclusiones de esta investigación posibles de enunciar son: la concepción de sujeto sobre la cual construye sus fundamentos de intervención la TO, está fuertemente influenciada por la concepción que el Estado tenga de los sujetos y cómo éste administra políticamente esas vidas. La TO es un instrumento del ejercicio biopolítico del Estado Chileno, es funcional a las fuerzas políticas que la configuran externamente. Durante la gubernamentalidad neoliberal se produce una irrupción en el desarrollo disciplinar, que produce deshistorización, silenciamiento y disciplinamiento al poder dictatorial.
\end{abstract}

Palabras Clave:

Gubernamentalidad, biopolítica, genealogía, dispositivo, fuerzas políticas.

\begin{abstract}
This research shows the relationship between socio-political aspects of the state, and Chile Occupational Therapy from a genealogical perspective of analysis, from this analysis is explained, how it reproduces this relationship in the knowledge and practices of Occupational Therapy (OT).

It is considered as a conceptual framework for analyzing the exercise of power, two concepts developed by the author Michel Foucault governmentality and biopolitics. The OT in this context, is defined as a disciplining device, corresponding to an instrument of governance of the above.

The methodology corresponds to a genealogical analysis. An analysis of speech from a Foucauldian perspective, from 5 semistructured interviews to Occupational Therapists, jointly analyzed under the same methodology, historical texts of the discipline in Chile. The unit of analysis was the political.
\end{abstract}

1 Terapeuta Ocupacional, Universidad de Chile. Licenciada en Ciencias de la Ocupación. Magíster en Terapia Ocupacional, Universidad Andrés Bello. Docente Carrera de Terapia Ocupacional Universidad Autónoma de Chile. Fono: 89004870, correo electrónico: marcehs@vtr.net

2 Terapeuta Ocupacional, Universidad de Chile. Licenciado en Ciencias de la Ocupación. Magíster en Terapia Ocupacional Universidad Andrés Bello. Director de Carrera de Terapia Ocupacional Universidad Andrés Bello, sede Concepción. Fono: 041-2662055, correo electrónico: cvalderrama@unab.cl 
The findings of this research are possible to state: The conception of the subject on which he builds the foundations of OT intervention, is strongly influenced by the conception that the State has of the subject and how it manages those lives politically. The TO is an instrument of the Chilean state biopolitical exercise, functional to the political forces that shape externally. Neoliberal governmentality During an eruption occurs in the development of the discipline, which produces dehistorization , mute and discipline to dictatorial power.

Keywords:

Governmentality, Biopolitics, Genealogy, Device, Political forces

\section{INTRODUCCIÓN}

El siguiente artículo corresponde a un extracto de la investigación Genealogía de un dispositivo: Historia Crítica de la Terapia Ocupacional en Chile desarrollada por los autores, en el marco de su tesis para optar al grado de Magíster en Terapia Ocupacional. Dicha investigación analiza desde una perspectiva genealógica foucaultiana, la procedencia, la emergencia, las fuerzas políticas y la subjetivación que han constituido a la Terapia Ocupacional en Chile.

Para efectos de este trabajo, se ha seleccionado la categoría de Fuerzas Políticas debido a la relevancia histórica nacional, en el marco de la conmemoración de los 40 años del Golpe de Estado y los 50 años de historia de nuestra disciplina en Chile, aportando con una mirada dirigida a visibilizar cómo las condicionantes sociohistóricas producen saberes y prácticas al interior de la Terapia Ocupacional desde su génesis hasta el fin de la dictadura militar.

Dado que se opta por una perspectiva foucaultiana de análisis, es necesario definir el marco teórico desde el cual se sustenta esta investigación. Para ello, se describe el concepto de dispositivo aportado por Foucault y explicado por Moro (2006) y los conceptos vinculados al ejercicio del poder: Biopolítica y Gubernamentalidad (Foucault, 2009), los cuales permiten comprender la relación entre las fuerzas políticas gobernantes y nuestra disciplina.

Comenzaremos entendiendo la Terapia Ocupacional como un Dispositivo, pues Moro (2006) señala tres aspectos fundamentales que constituyen este concepto. Primero, el dispositivo remite a una interacción de un conjunto heterogéneo de elementos como discursos, prácticas, normas, conceptos, leyes, instituciones, literatura, entre otros (nuestra disciplina presenta su propio discurso, saber y práctica). Segundo, el dispositivo permite develar las relaciones de poder ocultas bajo la constitución de un campo del saber, es aquel espacio donde se entremezclan y cruzan los discursos cargados de verdad y los mecanismos, prácticas e instituciones de poder (la Terapia Ocupacional se inscribe sometida al poder médico). Y en tercer lugar, el dispositivo define procesos de subjetivación ligados a la constitución de un saber y al ejercicio de algunas relaciones de poder (la Terapia Ocupacional produce subjetivación en los sujetos de intervención y en los propios cuerpos disciplinares, los terapeutas ocupacionales).

Con respecto a la Biopolítica, Foucault (2002) analiza que a partir de la segunda mitad del siglo XVIII, se instala esta tecnología que abarca la totalidad de la población desde un nivel de control macro: no sólo los individuos van a ser vigilados y transformados en sujetos dóciles a través del continuum disciplinario (el cual atañe al hombre - cuerpo) para el ejercicio del biopoder, sino que también la población en su conjunto va a ser estudiada y determinada a través de políticas globales y estandarizaciones que concibe al hombre - especie, es decir, estrategias biopolíticas.

En palabras de Foucault (1976, citado en Castro, 2008), la biopolítica, es entendida como la tecnología que permite que

el cuerpo se halle transido por la mecánica de lo viviente y que sirve de soporte a los procesos biológicos: la proliferación, los nacimientos y la mortalidad, el nivel de salud, la duración de la vida y la longevidad, con todas las condiciones que puedan hacerlos variar (p. 324).

Es la introducción de lo biológico lo que permite ejercer un dominio de la población y lograr la regularización de esta misma a través de las políticas estatales, las cuales comienzan a basarse en medidas estadísticas y, por tanto, abstractas, que invisibilizan a los sujetos, tornándose más simple la gubernamentalidad (Castro, 2008).

La gubernamentalidad según Foucault (2009), corresponde a las técnicas de gobierno que sirven de base a la formación del Estado, establece que ésta es "la ma- 
nera como se conduce la conducta de los hombres" (p. 448). Rose (1997) distingue la gubernamentalidad liberal que consiste en una nueva relación entre gobierno y conocimiento: en este ámbito se establece que el conocimiento positivista de las conductas humanas y sociales gira en torno a una serie de dispositivos destinados a la producción, circulación, acumulación y legitimación de la verdad, con el objeto de convertir en dóciles aquellos ámbitos sobre los que el gobierno debe ser ejercido. Entiende a los sujetos gobernados como partícipes en su propio gobierno; supone que los individuos asumirán libremente las decisiones para cuidar sus vidas y al mismo tiempo se instalan una serie de restricciones y normativas que se asumen por medio de nuevos discursos y promesas. Además, para el gobierno liberal, está la preocupación permanente por evaluar lo realizado, cuestionar los procedimientos, diagnosticar los errores con la intención de gobernar mejor. Y existe la gubernamentalidad neoliberal (García de la Huerta, 2010) que reduce la libertad política a la libertad económica y sustituye al ciudadano por el labrador/consumidor. El mercado se convierte en una nueva forma de poder sustentado por el poder político y jurídico, se legitima la economía como eje conductor y constructor del modo de vida, y lo político y jurídico el sostén necesario para que sea posible. De esta forma el Estado no es destruido por el libre mercado y la economía, en la medida en que se ajuste y se acomode para que las reglas del mercado sean posibles y efectivas.

Por tanto, la biopolítica se constituye como una tecnología del gobierno de las poblaciones, como una expresión de la gubernamentalidad, cuando el interés de los gobiernos recae sobre la Higiene Social, es decir, sobre la preocupación de una técnica general sobre la salud, cuyo objeto es el bienestar de la sociedad. Para ello es necesario la generación y aplicación de un saber médico administrativo funcional a la administración del Estado, identificando y controlando los elementos asociados a la salud colectiva, a la salud de las poblaciones como la locura, las tasas de mortalidad y natalidad, las enfermedades más prevalentes y más costosas, las enfermedades más discapacitantes para el individuo, la familia y la sociedad. Así como todos aquellos aspectos que puedan significar un riesgo al orden social, cuya estabilidad conduce al progreso; la idea es mantener todo lo más normal posible para que los flujos de seres humanos y de bienes del mercado se desarrollen libremente y sin obstáculos. A partir de esta situación los gobiernos deben "tomar todos los medios de protección de los individuos y las colectividades para preservar a la raza humana de todas las causas de degeneración física y moral" (Murillo, 2010, p. 47).

Murillo (2010) entrega una noción que permite aclarar la relación entre biopolítica y gubernamentalidad, señalando por una parte, que la biopolítica hace referencia a una concepción de la sociedad, del Estado y la política en términos biológicos, especialmente en lo patológico. Supone que el Estado es un organismo en continuo desorden, la política debe basarse en lo patológico. Por otro lado y simultáneamente la biopolítica es utilizada para dar cuentas del modo en que el Estado, el gobierno se hace cargo, en sus cálculos y mecanismos, de la vida biológica del hombre.

Foucault (2009) señala que ambos ejercicios del poder, biopolítica y gubernamentalidad, van dirigidos a los individuos, a las colectividades, a las poblaciones.

\section{Metodología}

Se escoge la perspectiva Genealógica (Foucault, s.f.) como metodología, pues permite develar la Genealogía del dispositivo Terapia Ocupacional en Chile, pues esta última define procesos de subjetivación ligados a la constitución de un saber y al ejercicio de relaciones de poder.

La genealogía tal y como fue formulada por Nietzsche puede definirse en dos sentidos: en primer lugar, como una historia crítica o estrategia de desfundamentación de algunos valores sobre los que se construye nuestro presente... y en segundo lugar, la genealogía se define como una historia eficaz que pretende jugar un papel fundamental en el diagnóstico de la actualidad (Moro, 2006, p. 58-59).

La genealogía no intenta reconstruir la historia en forma lineal o buscar el origen de un fenómeno, sino que pretende escudriñar las condiciones desde las cuales se produce el conocimiento y un discurso científico como tal; estudia la formación dispersa y discontinua de un discurso, aspirando a dilucidar su poder de constituir dominios de objetos (Foucault, 2008).

Los ejes para develar esta genealogía son la procedencia, que "remueve aquello que se percibía inmóvil, fragmenta lo que se pensaba unido; muestra la heterogeneidad de aquello que se imaginaba conforme a sí mismo" (Foucault, s.f., p. 3). La emergencia, entendiéndose como la lucha de fuerzas que permiten la identidad del dispositivo Terapia Ocupacional; las fuerzas políticas, que producen continuidades o discontinuida- 
des al interior del dispositivo y que muchas veces se relegan al olvido, y la subjetivación, pues el dispositivo produce subjetividad en el sujeto interventor e intervenido por la Terapia Ocupacional (Castro, 2008).

Con este propósito, se realiza un análisis de discurso desde una perspectiva foucaultiana. Según Foucault (2009) en los discursos se encuentra implícita la formación de modalidades discursivas, ya que son conformados a partir de instituciones que los emiten. Se considera a la Terapia Ocupacional como institución (Guajardo, 2011), por tanto, conocer y analizar sus discursos corresponde a un acceso primordial para la emergencia de la categoría que se analizará: las fuerzas políticas.

Se elaboró una entrevista semiestructurada que permita conocer por medio del discurso de los profesionales las conceptualizaciones presentes en torno a las fuerzas políticas y los significados de éstos en torno al lugar que ocupaba la Terapia Ocupacional en esta dinámica. La entrevista está diseñada para conocer la visión y la relación que establecen los entrevistados entre la situación social, política, económica del país y la Terapia Ocupacional en Chile.

Las fuentes primarias de información fueron $5 \mathrm{Te}$ rapeutas Ocupacionales. El perfil de los profesionales comprendió los siguientes criterios: haber egresado de la carrera dentro de los periodos señalados (1963 y 1989), haber ejercido o estar ejerciendo la profesión por al menos 10 años, en cualquiera de los campos de acción en los cuales interviene la Terapia Ocupacional, extendiéndose inclusive al ámbito académico.

Los entrevistados se categorizaron por año de egreso, para la presentación de este artículo, de la siguiente forma:

- Entrevistado 1: 1966

- $\quad$ Entrevistado 2: 1974

- $\quad$ Entrevistado 3: 1976

- Entrevistado 4: 1982

- $\quad$ Entrevistado 5: 1989

Las fuentes secundarias de análisis fueron documentos y archivos de orden nacional de la profesión disponible, entre los que podemos señalar:

- Primer decreto de la formación académica (1966)

- $\quad$ Cartas de la primera directora de carrera (1971)
- $\quad$ Primer plan de estudio (1966)

- Manuscritos de las clases de Terapia Ocupacional aportados voluntariamente por los entrevistados. (1970, 1971, 1973)

\section{Análisis}

Existen dos fuerzas políticas presentes en la producción de la disciplina en Chile, las cuales nominaremos como la predictadura de orden liberal social demócrata, que se denominó Gubernamentalidad Liberal (Rose, 1997) y dictadura militar de orden de derecha neoliberal y militarizada, que se denominó Gubernamentalidad Neoliberal (De las Rosas, 2010).

\subsection{Gubernamentalidad Liberal}

Para efectos de esta investigación consideraremos este periodo el comprendido por los gobiernos de Eduardo Frei Montalva y Salvador Allende, aunque corresponden mundialmente a un periodo más extenso.

Es importante considerar el rol del Estado en la determinación de las condicionantes sociales en las cuales es producida la Terapia Ocupacional. En ese sentido, se define que el Estado chileno se regía y adoptó una política de bienestar social. Esta última es una de las trasformaciones más significativas experimentadas por la sociedad chilena entre 1924 y 1973, que consistió en el proceso de expansión de la influencia del Estado sobre las condiciones de vida de la población ${ }^{3}$. La expresión ideológica de estos Estados, apuntan hacia estrategias de índole más participativa e igualitaria.

En términos político-institucionales, el Estado va a potenciar una política social de carácter mixto, que favorece por un lado a la protección del trabajador asalariado y por otro a la creciente clase media. El Estado se ve enfrentado, por un lado, a las presiones derivadas de la creciente organización sindical, facilitada por la concentración obrera y por la importancia creciente de la industria, y por otro, a la consolidación de una cla-

\footnotetext{
Nota autores: lo que corresponde a una estrategia Biopolítica en el sentido de que los gobiernos liberales diseñaban una serie de estrategias para instalar en las subjetividades un modo de vida vinculado al autocuidado, a la salud y el control del riesgo de enfermar o de padecer una patología social, por medio del rol de la escuela, de la familia, de los hospitales.
} 
se media que significa un sector de consumo creciente (Memoria Chilena, s.f.).

Durante el mandato del Presidente Eduardo Frei Montalva (1964-1970) el gobierno incorpora la redistribución del ingreso como uno de sus objetivos prioritarios. Para alcanzar esta meta se expandieron los beneficios y se incorporó a sectores tradicionalmente marginados, como lo eran campesinos, sectores urbanos pobres y también los discapacitados. En la década de los sesenta, las políticas de superación de la pobreza se dan bajo un enfoque tradicional que se ocupa de áreas como la previsión social, la educación, la salud y la vivienda, con un fuerte énfasis en la provisión centralizada y en la entrega gratuita y general de los beneficios. El aumento del gasto estatal en servicios y prestaciones sociales generó toda una institucionalidad encargada de gestionar la política social a través de ministerios y servicios públicos como forma de enfrentar la problemática social (Olmos y Silva, s.f.).

En el periodo transcurrido de 1964 a 1973, el Estado fue el responsable de generar la oferta de los bienes y servicios básicos como educación, servicios sanitarios, sistema de salud y construcción de viviendas, y a la vez, incentivó la demanda de estos bienes y servicios mediante la concientización de la población acerca de la importancia social de extenderlos a las clases populares y sobre el derecho de la ciudadanía a demandar al Estado por su provisión. El Estado juega un rol integrador, que acoge las demandas sociales. Si bien no logra superar totalmente la pobreza ni las desigualdades, "instaló nociones de solidaridad, compromisos colectivos y proyectos compartidos, todo ello en el marco de un clima y sentimiento de amparo estatal" (Olmos y Silva, s.f., p. 5).

Algunos indicadores que dan cuenta de los avances sociales de este periodo son la disminución de la tasa de analfabetismo, extensión de la cobertura de la educación básica, profesionalización de la atención de salud, disminución de la mortalidad infantil, entre otros (Olmos y Silva, s.f.).

Los sujetos eran considerados seres eminentemente sociales, en la medida que la cooperación, la unidad y el bien común son el eje central del quehacer diario, como lo señala una de las entrevistadas al mencionar las características presentes en ese periodo y que fueron incorporadas en la formación académica: "en ese momento estaba muy abierto todo..., las instituciones, los organismos, había una apertura hacia afuera, hacia recibir extranjeros, hacia una unidad. Se hablaba mucho de la unidad con lo latinoamericano" (Entrevistada 1).
El punto de vista valórico en el cual la Terapia Ocupacional entiende un espacio de desarrollo, está en la dignidad humana ${ }^{4}$. La dignidad es un valor fundamental de una ética de la autonomía ${ }^{5}$. El valor de la dignidad lo entenderemos como parte de un movimiento, esencialmente histórico y por ello dinámico y cambiante (Rebellato, 2000). Esta conceptualización representa, una coherencia conceptual, en cuanto a las bases filosóficas de la Terapia Ocupacional, impregnadas por el Movimiento de Artes y Oficios (Conferencia Nacional de Directores de Escuelas Universitarias de Terapia Ocupacional, 2004), el cual, a su vez, emerge como una respuesta a la instalación de la industrialización.

La relevancia de esta relación entre el Estado de bienestar y la Terapia Ocupacional no es solo en términos ideológicos ${ }^{6}$, emotivos o de pertenencia, sino que el dispositivo efectivamente era parte de este entramado de relaciones sociales, era un actor más de estas relaciones de poder. De hecho su creación implicó un compromiso por parte del Estado de Chile, con organismos internacionales, en un convenio de colaboración mutua, que garantizara la continuidad en el tiempo, su desarrollo y praxis como lo refiere la siguiente frase:

Nosotros aparecimos porque además
estuvimos respaldadas por un organismo
potente, la OMS y la OPS, y que ellos
hicieron que, aquel lugar donde hubiese
una Terapeuta Ocupacional... que los
lugares donde hubiese una terapeuta
ocupacional, esos lugares iban a ser
abastecidos, habilitados y durante tres
años y si eso no funcionaba, porque la
terapeuta era mala, porque los directores
no le daban la posibilidad, iban a retirar
todo (Entrevistada 1).

4 Ser dignos es exigir el reconocimiento como sujetos de derecho, reencontrarse consigo mismo y con los otros, confiar en nuestras propias capacidades y potencialidades de vivir.

5 Autonomía que la Terapia Ocupacional supone como propósito fundamental fomentar en los sujetos de atención.

6 En el sentido de compartir una mirada, una perspectiva en común en torno a los sujetos, a lo social y el bienestar de los sujetos en sus acciones. 


\subsubsection{Inclusión de la Terapia Ocupacional en las políticas públicas de salud}

Además a lo anterior como fundamento para la generación de la Terapia Ocupacional, se plantea un diagnóstico realizado al inicio de los años 60 y de la consideración de la salud como un derecho de todos los habitantes del país como un factor determinante en el proceso de desarrollo. Se definió la política en este sector, encaminada principalmente a mejorar la cobertura y la calidad de las prestaciones de recuperación de la salud (Lavados, 1983).

Desde una perspectiva Biopolítica la Terapia Ocupacional es un dispositivo que permitiría cubrir y controlar a la población en situación de discapacidad, resolviendo problemas de rehabilitación y de recuperación de la salud. Lo anterior implica, que el Estado garantizara la apertura de puestos de trabajo para los terapeutas ocupacionales en formación y los ya formados, como lo señala una de las entrevistadas: "el SNS en ese tiempo contrataba una terapeuta ocupacional, que funcionara y que estuviera funcionando por bastante tiempo... y fue creada la ley, porque de hecho si tú averiguas, en cada hospital hay terapeutas ocupacionales a nivel de Chile" (Entrevistada 1).

Si bien como una estrategia Biopolítica la creación de la carrera estaba asegurada en el Estado Chileno a través de los compromisos adquiridos con organismos internacionales, esto se materializó con la participación de entidades gubernamentales, que eran administradas por el Estado, como la Universidad de Chile y su entramado de dispositivos constituidos por la Facultad de Medicina, la clínica psiquiátrica y el Hospital José Joaquín Aguirre, en conjunto con la participación del Servicio Nacional de Salud. Esta estrategia sugiere que no sólo se pensaba en la creación de la Terapia Ocupacional como un carrera universitaria, sino que fue articulada para garantizar la continuidad, desarrollo y aplicación de sus acciones en los individuos donde se requiriese, en cualquier lugar de Chile, "se lograba crear en el Servicio Nacional de Salud, cargos para todos los que egresaban, si egresaban 10, había 10 cargos" (Entrevistada 1).

Se establece una estrategia que posibilita al Estado, no sólo resolver un problema de salud, sino que también ejercer un control social, de gobernabilidad, en la medida que más alcance y distancia logra, mayor influencia sobre la vida de las poblaciones tiene (Foucault, 2009). Eso nos permite visibilizar las fuerzas políticas y los mecanismos de control y distribución de éstas, en la génesis de la Terapia Ocupacional en Chile.

\subsubsection{La Unidad Popular y la transformación del dispositivo}

Durante el gobierno de Salvador Allende en el año 1970, se realiza un diagnóstico de la situación de la salud, el cual revela el mayor énfasis puesto en la medicina reparativa y en la medicina social, destacando la existencia de déficit de atenciones preventivas y de rehabilitación (Lavados, 1983). En este contexto, se entiende las prácticas discursivas del cuerpo del dispositivo en torno a los elementos teóricos y relacionales que determinaban la concepción de lo social y que eran compartidos e internalizados en la disciplina, como lo señala una de las entrevistadas al referirse a los elementos valóricos compartidos por la sociedad chilena en ese periodo "porque había un sentimiento hacia la gente, con más derecho, más igualitario, como que yo me acuerdo que salíamos todos a marchar, salíamos todos con los médicos... había toda una cosa mucho más horizontal en todas la situaciones, no como ahora" (Entrevistada 1).

Es dentro de este marco socio-político, en un contexto nacional de reforma de la educación, que surgen los primeros intentos por definirse como una profesión, por reconocer debilidades e insuficiencias en la formación y exigir modificaciones a la malla curricular.

En el año 1971, en una iniciativa del centro de alumnos, en conjunto con la escuela de Terapia Ocupacional, cuya directora de ese entonces era la señorita Brígida Flores, convocan a la Primera Convención Nacional de Terapeutas Ocupacionales (Escobar \& Sepúlveda, 2003). A partir de esas jornadas, la Srta. Flores redacta el anteproyecto del plan de estudios para la carrera de Terapia Ocupacional al decano de la Facultad, en ese mismo año, algunas de estas conclusiones enuncian "que la formación del Terapeuta Ocupacional en nuestro país ofrecida en los primeros años limitaba al profesional en su quehacer, por ser tecnocratizante y tomada de modelos extranjeros no acordes con nuestra realidad". ${ }^{7}$

En este documento, se alude a la necesidad de formar un profesional que estuviera contextualizado y

\footnotetext{
Documentos Escritos Originales A, 1971, facilitados por un ex miembro del Colegio de Terapeutas Ocupacionales de Chile.
} 
situado en su realidad, y con nuevos conocimientos del trabajo comunitario, "saliéndose de los estrechos márgenes del hospital, expandiendo su radio de acción a la comunidad misma". ${ }^{8}$ El programa es aprobado en 1972, agregando un semestre a la formación, con asignaturas como antropología, psicología social, introducción al método científico y aumentando las horas del área profesional específica.

Las fuerzas políticas social demócratas, de centro izquierda y de izquierda, presentan las características que facilitan establecer a la Terapia Ocupacional como un dispositivo instalado en el aparato estatal, que se materializa con la incorporación masiva de terapeutas ocupacionales para que se desempeñen en el sistema público:

- Hay una mayor disposición de escuchar las demandas ciudadanas, entiende como el verdadero patrimonio de una nación a sus ciudadanos, y que el desarrollo del país está en directa relación con el desarrollo de éstos.

- La fuerza política de los movimientos sociales de trabajadores, pobres, vulnerados, profesionales, la ciudadanía en su conjunto, fortalecida para ejercer sus derechos, asume un rol participativo y protagónico que permite resolver los problemas de la cuestión social. (Memoria Chilena, s.f.)

\subsubsection{Unidad Popular, Biopolítica y Terapia Ocupacional}

En el Gobierno de Allende la disciplina tenía un carácter biopolítico disciplinante, sin embargo, la perspectiva de este carácter estaba orientado a la buena vida, al buen gobierno de sí. Se entendía la intervención y la acción de la disciplina centrada en los sujetos de atención, "... Atendiendo a la persona y preocupándose de la persona por completo, independiente que estén adentro de un sistema hospitalario" (Entrevistado 5), dando cuenta de que para la Terapia Ocupacional los sujetos son fundamentales en la relaciones, la prioridad no es la técnica, no es el saber ni el conocimiento como instrumento del poder, sino que la persona misma.

La expresión Biopolítica del dispositivo en el liberalismo estaría acorde a la estrategia gubernamental de

8 Documentos Escritos Originales A, 1971, facilitados por un ex miembro del Colegio de Terapeutas Ocupacionales de Chile. la Unidad Popular para gobernar, cuyas características son:

1. Legitimar a través de la acción de los expertos, bajo la consigna del rescate del sujeto, el quehacer de las disciplinas emergentes para el control de las poblaciones, y principalmente.

2. El rescate del sujeto en la acción frente a los problemas de salud y de la discapacidad en el sentido de que éstos aprendan a gobernarse y controlarse a sí mismos por medio de las actividades terapéuticas realizadas en Terapia Ocupacional: "son cosas que realmente, yo creo, van intrínsecas en el ser humano, al realizar cosas manuales y que está en uno" (Entrevistada 1).

3. Con la irrupción del golpe militar y la instalación de la economía neoliberal como eje del modo de vida de los chilenos, comenzaron una serie de transformaciones sociales, políticas, económicas que serán coherentes con una fuerza política de carácter autoritario y represivo representado por los partidos de derecha, el mundo civil mayoritariamente oligárquico y las fuerzas militares. En este escenario, la disciplina es trastocada y sufre una serie de transformaciones en su interior.

\subsection{Gubernamentalidad neoliberal}

García de la Huerta (2010), propone que el neoliberalismo sería un constructor de Estado, para ello, ejemplifica lo ocurrido en Alemania de 1945 y Chile de 1973, ya que en ambos casos tendrían ciertas analogías, por ejemplo, que ambas formas de gobernar fueron luego del derrocamiento de gobiernos precedentes. En ambos gobiernos se acudió a la economía como instrumento de legitimidad gubernamental en el sentido de que ésta se vuelve creadora de derecho público.

Considerando al mismo autor, el neoliberalismo se puede describir cómo la razón económica adquiere un carácter total, tiene una implicancia enorme en el ámbito de la gubernamentalidad, en el sentido de que a partir de su instalación, y el adecuado funcionamiento de la estrategia económica asociada a un crecimiento sostenido, una moneda fuerte, precios estables y una balanza de pagos equilibrada, los gobiernos serán considerados buenos. 


\subsubsection{La Imposición de un régimen neoliberal en Chile}

La inflación y los conflictos sociales que se produjeron durante la Unidad Popular, hicieron que se fortaleciera la idea en algunos sectores sociales -principalmente de derecha- de apoyar el advenimiento de un gobierno militar, ya que veían en ellos la oportunidad perfecta de avanzar hacia la instalación definitiva de una economía que respondiera al paradigma neoliberal (Olmos, Silva, s.f). Así, el 11 de septiembre de 1973, mediante un golpe de Estado conjurado por los grupos oligárquicos con intereses económicos, las Fuerzas Armadas y la ClA, se instauró un régimen militar, institucionalizado en una Junta de Gobierno encabezada por el Comandante en Jefe del Ejército, el General Augusto Pinochet.

En este periodo se produce una importante caída del gasto público social. Por una parte se lleva adelante con una política anti-inflacionaria, que se basa en una fuerte reducción de los gastos de gobierno. Por otra parte, la disminución de gastos es estimulada por una estrategia de desarrollo de largo plazo, que descansa fundamentalmente en el sector privado y que procura reducir al Estado a su mínima expresión. En las áreas de educación, salud, vivienda y previsión, se realizan reformas que apuntan hacia una mayor injerencia del sector privado y del mercado (Arellano, 1985).

Existen dos elementos propios de la dictadura militar, íntimamente vinculados; por un lado, la instalación del modelo neoliberal, y por otro lado, los mecanismos represivos que fueron utilizados para permitir la instalación del modelo. Aunque el análisis no pretende realizar una revisión exhaustiva en torno a estos elementos, sí serán considerados para entrelazar, develar y comprender cómo estos elementos son los enunciados que permiten las prácticas discursivas del dispositivo disciplinar, y al mismo tiempo, cómo el ejercicio del poder penetra, transforma, modifica, reprime y silencia la disciplina.

Esta fuerza política que configura la Terapia Ocupacional a partir del año 73, produce profundas continuidades - discontinuidades en la historia de la Terapia Ocupacional en Chile.

\subsubsection{Efectos en la Terapia Ocupacional: el silencio del dispositivo}

Con las medidas incorporadas a nivel de gobernabilidad, se puso en riesgo la garantía que existía hasta ese momento en torno a la disponibilidad de puestos de trabajo para los Terapeutas Ocupacionales que egresasen de la escuela. Se cerraron puestos de trabajo y no se volvieron a abrir, con el fundamento de que una vez estabilizado el país se podría reestudiar las necesidades de estos profesionales, "de hecho nosotros egresamos en enero del 73, en septiembre fue el golpe. De mi generación y de las generaciones anteriores quedaron muy poca gente trabajando, muy poco terapeutas, un gran porcentaje se fue fuera, de inmediato. No te daban trabajo, de hecho yo como había participado en el centro de alumnos y al primer trabajo que postulé fue al J.J. Aguirre, que de hecho uno de los médicos, de ahí fue que me llevó al hospital del... y no me dejaron ... entonces yo de ahí trabajé en audiometría, en otra área" (Entrevistada 2).

Se manifiesta que si bien la reducción de gasto fiscal era importante en relación a la posibilidad de disponer de un puesto de trabajo, los terapeutas ocupacionales reconocen y manifiestan la evidencia que este fenómeno también estaba fuertemente vinculado a las fuerzas políticas imperantes en este periodo. El ejercicio del poder por parte de la fuerza política militar de derecha, significó la retirada del país de parte del cuerpo del dispositivo Terapia Ocupacional. Luego de que algunos Terapeutas Ocupacionales retornaran, pues por motivos políticos debieron emigrar, las condiciones para ejercer la disciplina no habían mejorado, por lo tanto, buscaron nuevas alternativas para generar ingresos, especialmente aquellos identificados como partidarios de la Unidad Popular: "cuando volví acá, yo volví con mi hijo.; ya?, necesitaba trabajar... Un tiempo la carrera estuvo cerrada acá, no había cargos en salud, por tanto era súper difícil encontrar. No lo encontré, me puse a estudiar un secretariado y cuando terminé el secretariado y como no había nada, no había salido nada aún y me puse a trabajar como secretaria... 10 años más o menos, no trabajé hartos años" (Entrevistada 2).

Hubo consecuencias objetivas en torno al movimiento que se produce con las personas tanto que trabajaban como aquellos que estudiaban, la disciplina no queda al margen de la cultura del miedo instalada en la dictadura militar, "el problema fue que el año 73 se perdieron muchos vínculos, eso fue, realmente cuando uno lo analiza, lo piensa, realmente significó un quiebre muy grande" (Entrevistada 2). Silenciando al dispositivo luego de las posteriores medidas represivas de la dictadura "...y con las generaciones anteriores que alguna vez me encontré después en la calle... la gente que estaba trabajando como que se quedó... que 
se quedó en su espacio, tratando de no meter bulla, de no hacerse visible" (Entrevistada 2). Por otro lado, otros profesionales con vinculación partidista se mantuvieron movilizados en la medida de lo posible, como lo señala una de las entrevistadas: "A pesar de todo yo igual me metía en cosas po', igual nos reuníamos los profesionales clandestinamente, nos reuníamos, me acuerdo que era en el Colegio de Asistentes Sociales" (Entrevistada 1), dando cuenta que al interior de la disciplina, se mantuvo un esbozo de la ideología inicial impregnada en su génesis.

Otro efecto que produjo la dictadura militar fue que los terapeutas comenzaron a desarrollar funciones ajenas, no propias de la disciplina, funciones lejanas y distantes a la relación con las personas, la idea aparentemente de control era evitar el contacto y las relaciones con los usuarios por parte del dispositivo, "cuando yo empecé a estudiar, en primero empecé a hacer ayudantía en fisiología y era en un laboratorio de neuropsicología acústica y ahí yo aprendí audiometría: Me entrenaron en el hospital, el j ${ }^{9}$ y en el Barros Luco. Las audiometrías era campo de nadie, no estaba incluido, que ahora está incluido en los tecnólogos médicos y había muy pocas fonoaudiólogas que habían estudiado afuera, porque no existía aquí la carrera de Fonoaudiología" (Entrevistada 2).

En otras ocasiones los Terapeutas Ocupacionales eran enviados a realizar sus funciones a lugares excluidos, a espacios y grupos olvidados por el Estado, ya que no eran prioridad en la reconstrucción económica planificada, eran los grupos considerados de alto riesgo y conflictivos, gobernados por un desamparo no solo vivido en el cuerpo del dispositivo, sino que en los grupos sociales vulnerados, como lo señala una de las entrevistadas al relatar su experiencia laboral en esos años: "me mandaron a hacer unas tareas en la San Gregorio, me mandaban así a terreno, sin ningún apoyo, sin nada y ahí estuve trabajando un tiempo" (Entrevistada 1).

Por otra parte, las generaciones que se encontraban en formación al momento del golpe militar, también fueron afectadas por éste, en el sentido de que algunos estudiantes no pudieron continuar la carrera, porque tuvieron que abandonar el país "después del gran último paro y después del pronunciamiento militar, retomamos las clases, hubieron alumnos que no

9 El entrevistado se refiere al Hospital José Joaquín Aguirre, hoy Hospital Clínico de la Universidad de Chile.
Ilegaron, y no llegaron porque varios se fueron del país" (Entrevistado 3). Y quienes permanecían estudiando no comentaban lo acontecido "ni menos lo ibas a tocar en una época de pronunciamiento militar y toda la huifa que pasó, no los tocabas, no se tocaban esos temas" (Entrevistado 3).

El golpe militar produjo como efecto inmediato el desmembramiento de la disciplina, entre lo que podemos señalar, se encuentra la destitución de la Directora de la Carrera Brígida Flores, la retirada de estudiantes partidarios de los partidos políticos pertenecientes o que apoyaron la Unidad Popular.

Además podemos señalar el cambio de funciones laborales como forma de castigo para los Terapeutas Ocupacionales en el ejercicio de sus funciones, el silencio y la clandestinidad de profesionales egresados predictadura que compartían los principios y valores de la Unidad Popular, los cuales fueron interiorizados durante su formación profesional, pero que se encontraban en el ejercicio de la disciplina en espacios controlados por la institucionalidad de la dictadura militar.

Lo mismo ocurrió en torno a la capacidad de reflexión y de espacios de discusión que pudiesen tener los estudiantes durante su formación profesional, en relación a los eventos políticos y sociales que ocurrían en ese periodo y las repercusiones que éstos acarrearían, posteriormente al golpe militar: "mira curiosamente había ingresado al segundo año del golpe militar a la universidad, yo creo que en ese momento el tema político como tal era un tema que estaba como aturdido, escondido, anestesiado y obviamente por el temor que implicaba el poder abrazar o declarar cualquier corriente política que fuera distinta a la imperante en el momento" (Entrevistado 4).

El miedo, como cultura de dominación que comenzó a gestarse a principios de los años 70, se materializa durante la dictadura militar, como señala el Programa de Naciones Unidas para el Desarrollo [PNUD] (1998, citado en Pincheira, 2010): "en los años 70 y 80 la sociedad chilena se encuentra dominada por una 'verdadera cultura del miedo': miedo al comunista, miedo al subversivo, miedo al cáncer invisible que corroe al cuerpo social. Miedo a la represión y al delator, miedo a ser "descubierto" en alguna (no se sabe cuál) imprudencia" (p. 129).

Esta situación produce sujetos desconfiados, altos grados de incertidumbre y frustración individual, miedo al otro, inseguridades, ensimismarse en el mundo privado, competencia para sobrellevar la adversidad (Pincheira, 2010), guardar silencio como efecto final. 
Al respecto, "el tema de lo represivo que imperaba en el momento no era un tema ni de discusión, ni ideas que se plantearan en una conversación abierta y menos desde el punto de vista académico porque en realidad era meterse, yo creo, en camisa de once varas. Por lo tanto, yo creo que nosotros estuvimos en un momento histórico político, pero que fue un momento de silencio" (Entrevistado 4).

Si la cultura del miedo a través de la represión, es capaz de inmovilizar a casi la totalidad de una nación, por supuesto que va a producir diversos cambios en el dispositivo disciplinar, que además sólo contaba con diez años de historia en nuestro país. Esta fuerza política hegemónica, dominante y autoritaria de la dictadura militar re-produce al dispositivo, garantiza la incapacidad de movimiento del mismo, de resistencia, lo obliga a volcarse hacia dentro, a centrarse en ámbitos netamente conceptuales, académicos y del hacer efectivo y competente, desarrollándose principalmente en ámbitos predominantemente clínicos. El dispositivo es forzado a transformarse en un espacio de relaciones apolíticas, ahistóricas, obedientes, irreflexivas, dessituadas y acríticas, y lo relega a los espacios olvidados, bajo una fuerte vigilancia.

Considerando la conceptualización de las personas y la ideología imperante en la época de formación de la disciplina, establecemos que la Terapia Ocupacional podría representar un riesgo, una fisura menor y desconocida al sistema hegemónico que se estaba instalando en Chile a partir del año 1973 “...en ese momento para la escuela de medicina de la Chile habían... muchos movimientos de distintos tipos, que eran clandestinos y Terapia Ocupacional en la Escuela estaba gente muy movida, como que la escuela siempre fue movida en ese sentido político...la Escuela estaba en la Facultad de Medicina Norte, en ese tiempo, y esa facultad era bastante roja..." (Entrevistada 5).

La Terapia Ocupacional intentaba no renunciar a sus principios de colectividad, de dignidad, de derechos y participación, sin embargo, el contexto, y la estrategia sistemática del ejercicio de poder utilizada por el Estado sometió al dispositivo, lo cooptó y fue subjetivado por la fuerza política instalada bajo la dictadura.

Se vivencia la capacidad de control del aparato del Estado sobre la disciplina y los dispositivos humanos que estaban involucrados en la vigilancia, control y ejecución de este ejercicio del poder, "... nosotros no tuvimos graduación por ej, porque... la directora dijo que no porque nosotros nos portábamos mal, porque nosotros participábamos en las marchas" (Entrevistada
5), dando cuenta que la obediencia, la docilidad de los cuerpos fueron inscritas en el dispositivo para mantener el orden social.

Todas estas medidas y situaciones generadas a través del ejercicio del poder por parte de la dictadura militar en la Terapia Ocupacional, correspondió a una práctica sistemática y planificada, que duró varios años, los efectos que produjo en el dispositivo no son azarosos ni circunstanciales, son efectos profundos, que dejaron huellas en el cuerpo del dispositivo.

\subsubsection{Deshistorización}

Los hechos sistemáticos de revisión y vigilancia que continuaron sobre la Terapia Ocupacional fueron varios y se expresan de distintas maneras.

El cierre de la carrera durante dos años para revisar la malla curricular. No existe claridad al interior del dispositivo para explicarse este hecho y gran parte del mismo tampoco ha conocido de esta suspensión histórica. Este efecto es identificable, a través de las prácticas discursivas sobre la propia historia de la disciplina, se denota una falta de conocimiento sobre la propia historia de la disciplina, "mira, para serte honesto, creo que ninguno de nosotros, hizo ni preguntas, más en detalle, y si llegamos a preguntar no tuvimos respuesta de la razón por la cual las autoridades decidieron suspender la carrera por dos años" (Entrevistado 4), "yo insisto que, creo que no tenían idea de lo que era la Terapia Ocupacional, algo extraño y si es que lo pudieron haberlo entendido como algo diferente, porque no lo conocían" (Entrevistado 3), "Ignorancia, ignorancia no más, porque no lo conocían, yo creo que no tenían idea de lo que era la terapia ocupacional" (Entrevistada 4), "Mira, el porqué del 75 al 77 se cerró no lo sé, no lo he leído, ni me recuerdo haberlo escuchado, no lo sé..." (Entrevistada 5).

Se plantea una causa de orden político; una posible explicación reside en la revisión minuciosa del plan de estudios, ya que desde el cambio en el año 1973, existían asignaturas vinculadas a las ciencias sociales, al trabajo grupal y comunitario, vinculadas a la crítica, la movilidad social y el rescate del sujeto. Por otro lado, la gran cantidad de asignaturas ligadas a las artes manuales, que representaban importantes formas de expresión popular en dictadura.

Sin embargo, luego de dos años de interrupción, se vuelve a abrir la carrera en el año 1979, sin ninguna 
modificación, hasta el año 1981 donde se eliminan las asignaturas de antropología y sociología.

Entonces el dispositivo se transforma en una disciplina deshistorizada porque establece estos hechos como una simple falta de conocimiento, sin comprender las relaciones de poder que estaba atravesando la Terapia Ocupacional en aquel contexto político.

Establecidos los puntos anteriores abordaremos la expresión Biopolítica de la disciplina en un contexto socio-histórico y político represivo y autoritario.

\subsubsection{Golpe militar, Biopolítica y Terapia Ocupacional}

La estrategia del ejercicio del poder desarrollada por la fuerza política de la dictadura fue la de establecer al interior del dispositivo la premura por centrarse en el saber, en el saber neutral, el saber científico, el saber del experto: "creo que las personas que fueron parte en este proceso formativo, tuvieron que centrarse netamente en el tema académico" (Entrevistado 4).

Lo anterior estaba acompañado de la cultura del miedo que provocaba que el saber histórico, el saber popular o cualquier otro tipo de saber quedase al margen de la formación profesional; “... habiendo vivido por ejemplo un tema extremadamente politizado, extremadamente politizado en el año $72,73 \ldots$ y después prácticamente vivir en un contexto en donde no habían variedades, sino que estaba la opción que no era la opción sino la imposición", "si alguien tenía, a lo mejor, una tendencia, la escondía y no la declaraba" (Entrevistado 4).

Esta condición produjo una profunda fisura al interior del dispositivo que lo obligó a ensimismarse para legitimarse en las ciencias médicas, a través del desarrollo de un saber científico, desconociendo el saber práctico subjetivo ${ }^{10}$ inherente a la disciplina.

En este marco, durante la dictadura se acentúa un conflicto político, un conflicto de fuerzas políticas al interior de los espacios de desarrollo de la Terapia Ocupacional, como el ámbito de la salud. Durante la dictadura militar se perdieron varios puestos de trabajo para terapeutas ocupacionales, "Hubo mucha ocupación de puestos de trabajo, de cargos que le correspondían al

10 En este sentido, se plantea que la Terapia Ocupacional es una disciplina esencialmente del hacer, de la praxis, vinculada al rescate del sujeto, al rescate de la subjetividad como construcción social.
TO y por no existir y desconocer o no buscar, lo tomaron Psicólogos, Asistentes Sociales y Kinesiólogos, esa era nuestra gran competencia" (Entrevistada 5). Lo relevante de este análisis es comprender que en el dispositivo se instala la práctica de la competencia, la práctica del posicionamiento por el poder, por sobre el posicionamiento a través de la legitimidad, se instala la lógica del mercado, de depredar, de poseer espacios de trabajo, de desarrollo profesional. Situación que produce una discontinuidad en el dispositivo, una irrupción a las lógicas de cooperación, de solidaridad que impregnaron la disciplina en su génesis.

Más aún, esta competencia se agudiza y coloca en desventaja a la Terapia Ocupacional cuando uno de los elementos a considerar es la igualdad de condiciones, y en ese sentido la disciplina se encuentra en inferioridad en términos estructurales, ya que la duración de la carrera era menor que otras con las cuales compartía campo de acción: "Por intereses, por conflicto de intereses. Habían otras carreras que de alguna manera no querían que uno fuese creciendo, y quitaba campo a otras carreras... había intereses creados de otras carreras que de alguna manera hicieron que no nos dejaran ampliar la carrera, porque al pasar a 4 años pasábamos a ser profesionales y no técnicos. Como nosotros no entendíamos mucho la parte política del tema" (Entrevistada 2).

Esto último refleja, la ingenuidad de la disciplina en torno a las implicancias del contexto político que enmarca sus prácticas y saberes, situación identificable de los discursos del cuerpo disciplinar, es decir, de quienes constituyen la disciplina, los Terapeutas Ocupacionales.

\subsubsection{Prácticas del dispositivo Terapia Ocupacional}

Otro efecto vinculado fuertemente a la estrategia Biopolítica ${ }^{11}$, tuvo que ver con la impronta y el significado que le comenzó a dar la Terapia Ocupacional a sus prácticas, y a partir de éstas, el significado y la conceptualización que entra en juego en torno a la subjetividad, en el sistema neoliberal.

Esta situación es relevante, ya que podría llamar a confusión y a comprender que la Terapia Ocupacional, en estas condiciones, ha sido capaz de generar un saber por sí misma, independiente de las condiciones socio-

\footnotetext{
Se establece que la Terapia Ocupacional es en este marco, un instrumento para el ejercicio del control y poder biopolítico, que se modifica a sí mismo, bajo la coherencia que le demanda la praxis de este ejercicio.
} 
históricas en las que está inscrita. Se hace referencia al rol social de la disciplina durante la dictadura y especialmente relacionado a la instalación de un sistema económico neoliberal, que apostaba a la regulación y crecimiento económico por los mecanismos propios del mercado, y no a la regulación protectora del Estado ${ }^{12}$.

Los Terapeutas Ocupacionales comprenden que su práctica es para beneficiar a los sujetos, pero a aquellos sujetos que están inmersos en las relaciones de producción y que por algún motivo, están apartados temporalmente de esta dinámica y corren riesgo de quedar fuera, "no, eso era únicamente esta cosa, yo diría casi revolucionaria, en un país que estaba convulso en aquellos años, de tratar, entre comillas, de salvaguardar la integridad laboral del sujeto" (Entrevistado 3).

Los sujetos de atención sobre los cuales actúa la disciplina varían, "en ese sentido la Terapia Ocupacional integra estas actividades porque hay un contexto social histórico que está representado en nuestros usuarios, el tipo de usuario que ingresaba a la unidad de Terapia Ocupacional, era el carpintero, era el albañil" (Entrevistada 5).

El TO incorpora que su labor, su práctica es congruente con lo que alguna vez consideró como propio, la preocupación y exaltación del sujeto, la dignidad del sujeto, en la medida que los sujetos trabajen, resguardan su integridad y dignidad. Sin embargo, se podría enmascarar que esa práctica es para retornar a la cadena de producción neoliberal a los sujetos, y en ese acto, no se pudiese estar dignificando el sujeto, sino que se pudiese favorecer su alienación cuando se ocupa trabajando. Esto ocurrirá en la medida que los terapeutas ocupacionales no consideran las condiciones sociales, laborales en la cuales se desarrolla la práctica del trabajo. En ese sentido, las condiciones en el neoliberalismo se han modificado a favor del mercado y no del sujeto trabajador.

12 La TO es la encargada de recuperar la capacidad funcional y productiva de los sujetos, y aquí se establece una conceptualización distinta en cuanto al periodo anterior a la dictadura, ya que en ese periodo si bien estaba en juego la dignidad de los sujetos, ahora la categoría de sujetos estaría dada en la medida que éste forma parte de la cadena de relaciones de producción económica, en ese sentido el sujeto es esencialmente productivo, por ende la ocupación es entendida como trabajo.

\section{Conclusiones}

A través de este artículo, se muestra las fuerzas políticas que han estado presentes en la conformación de la Terapia Ocupacional en Chile, fuerzas que han configurado la disciplina por las relaciones de poder que han establecido con ella.

La Gubernamentalidad Liberal, es identificada por el dispositivo como una fuerza generadora de la disciplina, especialmente en el entendido de que la Terapia Ocupacional se relaciona con sujetos, con las personas y las comunidades, y estos sujetos son iguales en derechos, en participación, en autonomía, en dignidad. Previo a la dictadura militar, el ejercicio biopolítico de la disciplina fue comprendido como el rescate del sujeto y la necesidad de aportar al gobierno de sí mismo en un marco social de solidaridad, respeto, tolerancia a la diversidad y bien común.

Es decir, la relación que se establece entre las condiciones sociopolíticas generadas en un Estado de Bienestar, para con los sujetos que se contienen en él, es un argumento para basar la práctica de la Terapia Ocupacional, ya que en ambas se rescata la subjetividad, como una construcción social.

Sin embargo, políticamente a partir del año 73, en un Estado de características represivas se generaron subjetividades marcadas por el miedo, la desconfianza, el desarrollo de la individualidad por sobre la colectividad, la competitividad, la disminución de soporte social, la percepción de soledad, el silenciamiento ideológico. En resumen, sujetos despolitizados y deshistorizados, ya que a partir de ese momento lo ontológico del modo de vida sería la capacidad del sistema social de contar con sujetos consumidores/labradores que en la medida de que su fuerza productiva genere su propio bienestar se lograría una sociedad desarrollada.

El neoliberalismo implicó una apertura a la inversión privada, el rol del Estado se reduce en la regulación y participación de la economía. El gasto público en una primera instancia se retrae fuertemente, especialmente en lo relacionado a las políticas sociales; lo social se posterga para lograr la estabilidad económica nacional. Esto afecta profundamente la disciplina, ya que se pierde el respaldo del Estado en cuanto a la instalación en el sistema público de la Terapia Ocupacional, se comienzan a cerrar cargos y no se abren más cargos, se privatiza la salud, por tanto las prestaciones entregadas en este ámbito debían tener una relación equilibrada entre costo - efectividad, es decir, la terapia ocupacional es puesta en jaque por lo largo de sus 
intervenciones y lo lento de los resultados esperados. Sin embargo, por otro lado, se abre con más fuerza un nuevo espacio de práctica como son las mutuales de carácter privado, pero tremendamente necesario para revitalizar la economía recuperando la fuerza de trabajo de los accidentados.

El neoliberalismo, considerado por sus defensores como el rescate económico de una crisis del capitalismo, se materializa en la Terapia Ocupacional modificándola, refundándola en sus prácticas y saberes que la sustentan, ya que se debe dar respuesta a las nuevas necesidades de la sociedad. Entonces en esta nueva época histórica de Chile, importa abordar a los sujetos desde otras perspectivas, ampliar nuestra visión del ser humano, que como podría interpretar Foucault, estos cambios de pseudo-humanización de las prácticas profesionales continúan siendo estrategias y tecnologías para ejercer el dominio, el control social y la conformación de cuerpos-subjetividades dóciles. Podría constituirse en una posible explicación de que el cierre durante dos años de la carrera en la Facultad de Medicina, no sufrió ninguna modificación en su malla curricular, hasta el año 1981, donde se retira Sociología y Antropología del plan de estudios.

Probablemente estas asignaturas constituían una amenaza política, ya que las ciencias sociales tienden a rescatar a los sujetos, la humanidad como patrimonio cultural, por tanto se inscriben en una permanente reflexión de la historia, la misma historia que el neoliberalismo parodia e ignora, y desea que desaparezca.

Durante la Gubernamentalidad Neoliberal la Terapia Ocupacional se silencia, se vuelve acrítica, irreflexiva, deshistorizada, por una situación de poder en la cual está inscrita y en los cuales ha estado sometida, sin embargo, en ningún caso significan inmovilidad, un estado estático, por el contrario, han servido para establecer tiempos de reacción, de acomodo o de continuidad. A pesar de ello, hubo momentos en los cuales se pudo hablar de derechos humanos, en plena dictadura, y se realizó trabajo con las comunidades.

Un elemento distintivo y constituyente de la disciplina, es ser un instrumento biopolítico que desarrolla el ejercicio del Biopoder; el Estado chileno de entonces, representado por la dictadura militar, al igual que en la unidad popular, establece la disciplina desde el punto de vista biopolítico, pero con un sentido distinto, es decir, en ambos casos la Terapia Ocupacional es un instrumento para el control social, para la recuperación de la salud, para presentar alternativas a los modos de producción. Pero los grupos objetivos van cambiando, sin dejar de ocuparse con los que ya trabajaba, en la dictadura los sujetos de atención son los trabajadores que sufren accidentes y los sujetos con discapacidad que pueden ser requeridos en las relaciones de producción.

De este trabajo se desprende la necesidad de analizar la relación de las políticas públicas y la Terapia Ocupacional a contar de los gobiernos de la Concertación, incorporando las implicancias en los saberes y prácticas de la TO cuando ésta es incorporada a una serie de políticas sociales, que incluyen no solo al sector salud, sino educación, justicia y protección social.

Por último, a partir de esta investigación se propone la necesidad de analizar su propia fuerza política disciplinar gremial, cómo ésta se configura, qué significados tiene para el dispositivo y en qué medida se plantea un desafío para su re-articulación y reconocimiento.

\section{ReferenCias BibliográficAs}

Arellano, J. (1985). Políticas Sociales y Desarrollo 1924-1984. Santiago: Editorial Cieplan.

Castro, R. (2008). Foucault y el cuidado de la libertad, ética para un rostro de arena. Santiago: LOM Ediciones.

Conferencia Nacional de Directores de Escuelas Universitarias de Terapia Ocupacional (2004). Libro Blanco de la Diplomatura de Terapia Ocupacional. Zaragoza.

Escobar, P. y Sepúlveda, R. (2003). Escuela de Terapia Ocupacional Universidad de Chile: Aportes para una historia posible. Santiago: $\mathrm{ETOUCH}$, formato digital.

Foucault, M. (s.f.). Nietzsche, la genealogía y la historia. Extraído 12 de junio de 2012 desde http://proyectoconstitucion2011.uniandes.edu.co/recursos/docs/

Foucault, M. (2008). Vigilar y castigar, nacimiento de la prisión. Buenos Aires: Siglo Veintiuno Editores.

Foucault, M. (2008). El Orden del discurso. Buenos Aires. Tusquets Editores.

Foucault, M. (2009). Seguridad, territorio, población. Buenos Aires. Fondo de Cultura Económica.

Foucault, M. (2011). Nietzsche, Freud y Marx. Santiago: Espíritu Libertario.

García, M. (2010). Foucault y Neoliberalismo: una lectura crítica. En V. Lemm, Michel Foucault: Neoliberalismo y biopolítica. (p. 177 - 197). Santiago: Ediciones Diego Portales.

Guajardo, A. (2011). En Grupo Ocupación y Realización Humana. Ocupación: Sentido, Realización y Libertad. Diálogos ocupacionales en torno al Sujeto, la Sociedad y el Medio Ambiente. (pp. 13 -21). Bogotá: Universidad Nacional de Colombia. 
Lavados, I. (1983). Evolución de las políticas sociales en Chile 1964 - 1980. Santiago de Chile: Organización de Naciones Unidas [ONU].

Memoria Chilena (s.f.). El Estado de bienestar social 1924-1973. Extraído el 15 de junio de 2012 desde http://www.memoriachilena. cl/temas/index.asp?id_ut=estadodebinestarsocial1924-1973

Moro, O. (2006). La perspectiva genealógica de la historia. Santander: Servicio de Publicaciones de la Universidad de Cantabria.

Murillo, S. (2010). Biopolítica, ciencia y tecnología. En I. Cassigoli y M. Sobarzo, Biopolíticas del Sur (pp. 35 - 50). Santiago: Editorial Arcis.

Olmos S., Silva R. (s.f). El rol del Estado chileno en el desarrollo de las políticas de bienestar. Series Indagación. Revista Expansiva.

Pincheira, I. (2010). Del miedo y la inseguridad, a las luchas ético/ estéticas en el Chile de hoy. En I. Casigoli y M. Sobarzo, Biopolíticas del Sur (pp. 275 - 290). Santiago: Editorial Arcis.

Rebellato, J. (2000). Ética de la liberación. Montevideo: NordanComunidad.

Rose, N. (1997). El gobierno en las democracias liberales avanzadas: del liberalismo al neoliberalismo. Revista Archipiélago: cuadernos de crítica de la cultura, 29, 25-40. 